\section{E.M. Site A.C. Magnetic Field Sources, Surveys and Solutions Part 2: ACMF/EM Antipathy}

Curt R. Dunnam

Linear Research Associates, Trumansburg, NY

A.C. magnetic field (ACMF) sources and their EM interaction mechanisms are discussed in this installment, which continues our July/August series on preventing and resolving site magnetic field problems. This article carries forward the theme of a primer on the basic physical causes and effects of A.C. magnetic fields, with special emphasis on the manner in which such fields may affect site EM instrumentation. Experience has demonstrated that a basic understanding of the various aspects of ACMF sources will often prove helpful in identifying the sources which actually pose a threat to EM site operations.

Like most space in commercial buildings, EM sites are subject to simultaneous ACMF's from many sources external and internal to the site building itself. External sources may include power transmission lines, substations and local stepdown transformers. The major internal sources are more numerous and varied, but typically include vaulted transformers, highcurrent primary busses, ballasted lighting, and motor-driven apparatus such as chillers, ventilation blower and air compressors. Common site-related ACMF sources are illustrated in Figure 1:

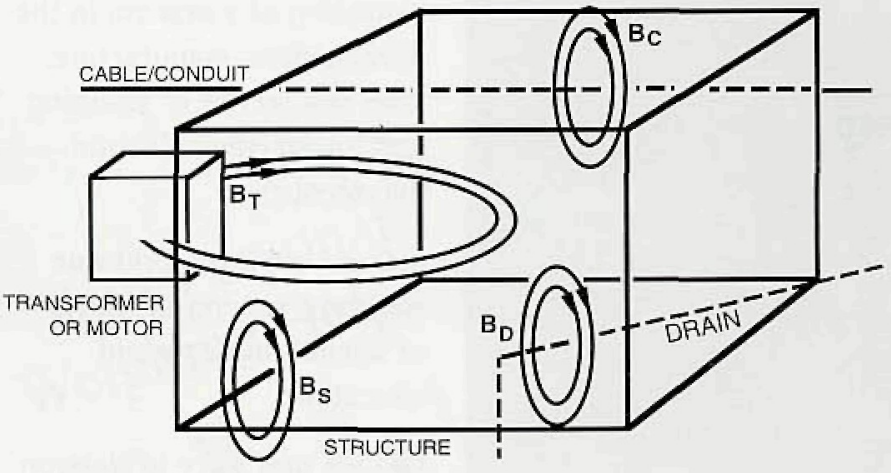

Figure 1: Typical EM Site A.C. Magnetic Field Sources

When estimating field influences at the EM location, it is useful to note that relatively high local fields from magnetic-core devices such as transformers and motors $\left(\mathrm{B}_{T}\right)$ decreases most quickly with distance, fields from high-current distribution busses $\left(B_{C}\right)$ decrease less rapicly and fields due to so-called ground currents through conductive paths such as the building structure $\left(\mathrm{B}_{S}\right)$ and metallic drain line $(\mathrm{s})\left(\mathrm{B}_{\mathrm{D}}\right)$ decreases the least rapidly of all (see Table 1).

Each alternating-current source produces a time-varying ACMF which can be characterized by peak strength, temporal waveform (which depends on harmonic content), orientation and magnitude-distance dependence. Typical site sources, ACMF values and dependence on radial distance " $r$ " are summarized in Table 1. In perusing the Table, you will see that the worst actors for high-valued proximate fields are devices such as transformers and motors. Coincidentally, these sources exhibit the highest rate of field decrease with added distance. This turns out to be a very fortunate state of affairs for EM users!

Using Table 1, it is fairly easy to make a rough determination of which sources might or might not pose a likely threat to a particular EM site. Such work requires only a measurement of the average ACMF value near any given field generator at which point its contribution to the actual or proposed
EM location ACMF may be estimated in a straightforward manner. This is accomplished by utilizing the distance dependence function for that type of source. For example, a transformer which produces a measured field of 100 milligauss ( $\mathrm{mG}$ ) at a distance of $1 \mathrm{~m}$ should produce only $0.5 \mathrm{mG}$ near an EM located $6 \mathrm{~m}$ distant. Any field generator estimated to produce in excess 0.7 $\mathrm{mG}_{\mathrm{rms}}$ at the EM location should be considered a problem source. Ground current loops, which exist throughout most buildings and exhibit a $1 / r$ dependence, commonly fall into this category.

DEPENDENCE ACMF, $\mathrm{mG}_{\mathrm{rms}}$

External Sources-Large

(Q) $15 \mathrm{~m} @ 30 \mathrm{~m}$
345 KV Transmission Line

$115 \mathrm{KV}$ Transmission Line

13.8 KV Local Transmission

5 MVA Substation

$$
\begin{gathered}
1 / \mathrm{r}^{2} \\
1 / \mathrm{r}^{2} \\
1 / \mathrm{r}^{1.5} \\
1 / \mathrm{r}^{3}
\end{gathered}
$$

Internal Sources-Moderate

20 KVA Distribution Transformer

480 V Distribution Buss

Internal Sources-Low

Fluorescent Fixtures

Compressor Motor

Ground currents (pipes, girders, electrical conduits, etc.)
$10 \quad 2$

$5 \quad 1$

$8<1.0$

(9) $5 \mathrm{~m} @ 10 \mathrm{~m}$

$1 / \mathrm{r}^{3} \quad 10 \quad 1.5$

$1 / \mathrm{r}^{2}$

5

@ $1 \mathrm{~m}$

12

8

3
$24-6$

\section{3}

(a) $2 \mathrm{~m}$

1.5

$<1.0$

1.5
Table 1: Estimated ACMF Source Field Magnitudes and Theoretical " $r$ " Dependence

At the EM, the resulting time-varying ACMF is determined by a vector summation over all individual source fields, although in practice only one or two source fields will be dominant. It is this summation ACMF vector which directly influences the EM instrument's charged particle beam. To understand the effect of the time-varying magnetic field on the EM instrument, it is necessary to understand the nature of three significant ACMF-EM interaction mechanisms.

All $A C M F$ interactions with an $E M$ are described by the $F=q[B \times v]$ Lorentz force you heard a lot about in undergraduate physics. By far the most prevalent of the three mechanisms is $E M$ transwerse beam displacement. Beam motion of this type is imparted by the ACMF vector component which lies in a plane normal to the beam axis. For vertical-column EM's this imaginary surface is horizontal and is universally defined to be the xy plane. Most EM's (both transmission and scanning) arrive from the factory exhibiting threshold $x y$ plane ACMF values of around $1.0 \mathrm{mG}_{\mathrm{rms}}$. The actual threshold value of course depends on beam energy (EM susceptibility is inversely proportional to its square root), EM internal shielding, ACMF harmonic content and mean path length over which a normal $\mathrm{ACMF}$ component exerts an influence on the beam.

Less frequently seen in practice is the EM beam defocusing effect which may be caused by a strong ACMF z-axis component. This influence is usually ascribed to distortion of the EM objective lens fields. Because focusing sensitivity is approximately an order of magnitude lower than the previously described transverse effect, the transverse effect usually dominates. Z-axis ACMF defocusing thresholds range from 10 to $15 \mathrm{mG}_{\mathrm{rms}}$ :

With the increasing use of field-emission (electron) guns (FEG's), a third ACMF interaction mechanism is more often observed which produces symptoms quire similar to the defocusing effect mentioned above. In such instances, FEG resolution loss may occur at isotropic ACMF threshold values in the vicinity of 1 $\mathrm{mG}_{\mathrm{rms}}$. The isotropic factor implies that both the emissive spot and subsequent electron trajectories are affected. ACMF components exceeding $0.5 \mathrm{mG}_{\mathrm{rms}}$ in any axis should be avoided for optimum FEG employment.

In this article in our ACMF series the point has been made that measurable ACMF's exist throughout buildings with energized A.C. distribution systems. But the relevant question is, do these inevitable ACMF's exceed $E M$ threshold limits at a given site? Armed with a basic knowledge of sources and EM interaction mechanisms, in the next article we will explore basic A.C. magnetic field survey data collection methods for meaningful site ACMF analysis. 